\title{
Concomitant Biceps Tenodesis Improves Outcomes for SLAP Repair: Minimum 2-Year Clinical Outcomes after SLAP II-IV Repair Versus Tenodesis Versus Both
}

\author{
T. J. Ridley, M.D., Marilee P. Horan, M.P.H., Philip-C. Nolte, M.D., M.A., \\ Bryant P. Elrick, M.D., M.Sc., and Peter J. Millett, M.D., M.Sc.
}

Purpose: To investigate clinical outcomes, return to sport, and complication rate in patients with an isolated SLAP II-IV tear treated with biceps tenodesis (BT), SLAP-repair (SLAP-R), or both (SLAP-R+BT). Methods: A retrospective analysis of prospectively collected data was performed in patients who underwent surgery between February 2006 and February 2018 for isolated SLAP II-IV lesions with either BT, SLAP-R, or SLAP-R+BT and had minimum 2-year follow-up. Patients were excluded if they were older than 45 years of age, had anterior shoulder instability, rotator cuff tears, glenohumeral osteoarthritis, or concomitant fractures about the shoulder. Clinical outcomes were assessed by the use of the American Shoulder and Elbow Society Score, Single Assessment Numerical Evaluation Score, Quick Disabilities of the Arm, and Shoulder and Hand Score, the General Health Short Form-12 Physical Component, and patient satisfaction. Results: There were 38 shoulders in the isolated BT group with $1(2.6 \%)$ shoulder requiring revision, 13 in the SLAP-R group with no patient requiring revision, and 21 in the SLAP-R+BT group with $2(9.5 \%)$ shoulders requiring revision. Minimum 2-year follow-up was obtained in $>85 \%$ of each group. Mean age at time of surgery was significantly different between the groups ( 36.5 years BT vs 27.7 years SLAP-R vs 36.5 years SLAP-R+BT; $P=.003$ ). While patient-reported outcomes improved significantly from pre- to postoperatively for the BT $(P<.001)$ and SLAP-R+BT groups $(P<.001)$, they did not significantly improve for the isolated SLAP-R group ( $P$ values ranging .635 to .123). The BT and SLAP-R+BT groups showed significant improvement in return to sport pre- to postoperatively whereas the SLAP-R group did not. The SLAP-R+BT group had the most patients reaching minimal clinical important difference, substantial clinical benefit, and patient acceptable symptom state American Shoulder and Elbow Society Score scores; however, this was not statistically significant. Conclusions: SLAP II-IV lesions treated with BT or both SLAP-R+BT demonstrated improved outcomes compared with isolated SLAP-R at minimum 2-year follow-up. Concomitant biceps tenodesis should be considered when performing repair of SLAP II-IV tears. Level of Evidence: III; Retrospective comparative study.

$\mathbf{S}^{\mathrm{T}}$ LAP tears may be a source of shoulder pain and disability in patients. Symptomatic type I SLAP lesions, characterized by fraying of the superior labrum with maintained attachment to the glenoid, are commonly treated with arthroscopic debridement. Type II-IV SLAP lesions involve varying degrees of labral detachment from the superior glenoid and can result in

From the Steadman Philippon Research Institute (T.J.R., M.P.H., P.-C.N., B.P.E., P.J.M.); and The Steadman Clinic (T.J.R., P.J.M.), Vail, Colorado, U.S.A.

The authors report the following potential conflicts of interest or sources of funding: T.J.R., M.P.H., and B.P.E. report other from Arthrex, Smith $\theta$ Nephew, Siemens, and Össur, outside the submitted work. P.-C.N. reports nonfinancial support from Arthrex, during the conduct of the study, and personal fees from Arthrex, outside the submitted work. P.J.M. reports grants, personal fees, and other from Arthrex; other from Smith $\theta$ Nephew, Siemens, and Össur; personal fees from MediBridge and Springer Publishing; and other from VuMedi, outside the submitted work. Full ICMJE author disclosure forms are available for this article online, as supplementary material. increased translation and shear forces of the glenohumeral joint. ${ }^{1,2}$ Surgical treatment options mainly include primary SLAP repair (SLAP-R), biceps tenotomy, or biceps tenodesis (BT).

Controversy remains over the optimal treatment for patients with symptomatic type II-IV lesions. Studies have demonstrated equivalent outcomes of SLAP-R

Research performed at the Steadman Philippon Research Institute, Vail, Colorado, U.S.A.

Received November 9, 2020; accepted October 11, 2021.

Address correspondence to Peter J. Millett, M.D., M.Sc., Center for Outcomes-based Orthopaedic Research, Steadman Philippon Research Institute, 181 West Meadow Dr., Suite 1000,Vail, CO 81657.E-mail:drmillett@ thesteadmanclinic.com

(C) 2021 THE AUTHORS. Published by Elsevier Inc. on behalf of the Arthroscopy Association of North America. This is an open access article under the CC BY-NC-ND license (http://creativecommons.org/licenses/by-nc-nd/4.0/). 2666-061X/201841

https://doi.org/10.1016/j.asmr.2021.10.007 
and BT, whereas others have suggested worse patient outcomes and return to sport following isolated SLAP-R compared with BT. ${ }^{3-5}$ A recent meta-analysis revealed that BT of patients in their 30s to 40s resulted in greater rates of patient satisfaction and return to sport rates compared with SLAP-R. ${ }^{6}$ However, return to play in high-level athletes and those younger than 30 years was improved with SLAP-R compared with BT, and caution remains regarding performing $\mathrm{BT}$ in overheadthrowing athletes. ${ }^{7,8}$ Currently, isolated SLAP-R is typically reserved for young patients or high-level overhead athletes with SLAP II-IV tears, whereas BT is reserved for patients older than 30 years and recreational-level athletes. ${ }^{6-8}$ While typically performed in isolation, neither has provided consistent results.

When BT is performed, concomitant SLAP-R is not typically performed. ${ }^{3,4}$ SLAP tears have demonstrated increased humeral head translation compared with biceps tenotomy alone, suggesting a role of the superior labrum in glenohumeral joint stability independent of the long head of the biceps. ${ }^{2,9}$ Based on this, some surgeons have suggested performing SLAP-R in addition to a BT, particularly in greater-demand patients in whom glenohumeral joint stability is of concern. However, an analysis of combined repair and tenodesis in high-demand patients demonstrated worse outcomes than those with repair or tenodesis performed in isolation. ${ }^{10}$ This study included a high proportion of overhead-throwing athletes $(70 \%-73 \%)$ with a significantly older population in the isolated BT group. In addition, there were a significant number of worker's compensation patients within the combined group, which may have led to worse outcomes in this group. Given these limitations, as well as the paucity of literature regarding combined SLAP-R and BT, further information is needed to evaluate the efficacy of combining these surgical treatments in patients with isolated symptomatic SLAP II-IV tears.

The purpose of this study was to investigate clinical outcomes, return to sport, and complication rate in patients with an isolated SLAP II-IV tear who were treated with SLAP-R, BT, or both (SLAP-R+BT). It was hypothesized that SLAP II-IV lesions treated with BT or both SLAP-R+BT would demonstrate improved outcomes compared with isolated SLAP-R at a minimum 2-year follow-up.

\section{Methods}

Following approval by the institutional review board (Vail IRB 2018-69), this retrospective comparative study was performed on prospectively collected data. Inclusion criteria were all patients between the ages of 14 and 45 years at the time of surgery and were a minimum 2 years out from surgery. Surgeries were performed between February 2006 and February 2018 for an isolated symptomatic SLAP II-IV lesion with either SLAP-R, BT, or SLAP-R+BT. All patients were treated by a single surgeon (P.J.M.). Patients were excluded if they were older than the age of 45 years, had anterior shoulder instability, rotator cuff tears, presence of glenohumeral osteoarthritis, or concomitant fractures about the shoulder.

Surgical management was indicated for persistent pain, loss of strength, and impaired function of the affected arm that did not resolve with physical therapy for at least 3 months. Patients also were included if they did not respond to previous arthroscopic debridement and/or previous SLAP repair. Isolated BT was indicated for patients with SLAP II-IV tears with primary complaints and findings regarding biceps tenosynovitis. These clinical findings included anterior shoulder pain, tenderness along the long head of the biceps, positive Speed's and Yergason's tests, or direct arthroscopic visualization of tenosynovitis. SLAP-R alone was indicated for those with isolated SLAP II-IV tears without clinical or operative findings of biceps tenosynovitis. SLAP-R+BT was performed in those patients with positive biceps findings with concomitant SLAP II-IV on intraoperative findings.

All of the following procedures were performed with the patient in the beach-chair position using an arm positioner (SPIDER; Smith \& Nephew, Memphis, TN). In all patients, an examination under anesthesia followed by the respective procedure was performed by the senior author (P.J.M.).

\section{Arthroscopic SLAP-R Technique}

Diagnostic arthroscopy was performed using standard posterior and rotator interval mid-glenoid portals and a thorough evaluation of the SLAP and bicipital root was performed to classify and confirm the type of labral tear. Following confirmation of an unstable superior labrum and classification of SLAP tear, II-IV, according to Snyder et al., ${ }^{11}$ the superior glenoid was debrided of soft tissue and minimal decortication was performed to reveal bleeding bony surface for improved healing capacity. An accessory anterosuperolateral portal was created through the rotator interval and secured by a $5-\mathrm{mm}$ cannula. In all cases, 2 knotless anchors (Arthrex, Naples, FL) were placed. The first one was placed via a curved drilling and insertion guide just anterior to the biceps tendon at the 1-o'clock position (right shoulder) on the apex of the glenoid rim while the shoulder was externally rotated. A curved arthroscopic sutureshuttling device was passed through the standard midglenoid portal and around the superior labrum. Care was taken to not include any additional capsular tissue when shuttling the suture around the labrum. The repair suture of the anchor was shuttled through the midglenoid portal and then passed back through the anterosuperolateral portal. The repair suture was placed into the shuttling suture and shuttled back through the 
self-locking mechanism of the anchor. The repair was then tensioned appropriately to fixate the anterosuperior labrum and the suture tail was cut flush.

Similar steps were performed to place the second anchor at the 11-o'clock position on the apex of the glenoid rim with a second knotless suture anchor (Arthrex). To shuttle the suture through the superiorposterior labrum, a spinal needle was placed in the location of the Neviaser portal. After visualization of both the tip of the needle below and the shaft of the needle superior to the biceps-labral complex, a \#2 PDS was placed into the needle and into the joint. The PDS suture acted as a shuttling suture passing the repair suture of the knotless all-suture anchor under the labrum and through the superior capsule. The repair suture was shuttled around the labrum and was retrieved above the labrum back out through the anterosuperolateral portal. The repair was finalized and tensioned in the same fashion as mentioned for the knotless, all-suture anchor. Care was taken not to entrap the biceps anchor, and the arm was placed in $30^{\circ}$ of external rotation before tensioning the 11-o'clock anchor to avoid overtightening the shoulder.

Postsurgery, the patient was maintained in a sling for 4 weeks. Full passive range of motion was allowed as tolerated. Biceps loading was avoided. At 4 weeks postoperatively, the patient began full active range of motion, and at 6 weeks the patient progressed to resistance training. Once full strength had been restored and the patient was pain free-typically after 4-5 months-unrestricted return to sports was permitted.

\section{BT Technique}

Similarly, a posterior and standard rotator interval mid-glenoid portal was created, and a thorough arthroscopic evaluation of the SLAP and bicipital root was performed to classify and confirm the type of labral tear. Next, a tenotomy of the long head of the biceps tendon (LHBT) was performed directly at the superior labral insertion and the SLAP region was debrided using a radiofrequency ablation device.

A subpectoral BT was performed in all cases of BT. Next, the arm was positioned in $90^{\circ}$ of abduction and $90^{\circ}$ of elbow flexion with the volar aspect of the forearm pointed downward and parallel to the floor. An incision was made extending from approximately $1 \mathrm{~cm}$ superior to $2 \mathrm{~cm}$ inferior to the inferior border of the pectoralis major tendon in line of the axillary crease. The pectoralis major tendon and the short head of the biceps tendon was identified. The fascia overlying the coracobrachialis muscle and short head of the biceps brachii was incised. Blunt dissection lead directly to the LHBT. We visually marked the tendon at its native resting length and a right-angle clamp was then used to retrieve the LHBT. The tendon was whipstiched with No. 2 nonabsorbable high-strength suture (FiberWire;
Arthrex), beginning $2 \mathrm{~cm}$ proximal of the musculotendinous junction so that $10-12 \mathrm{~mm}$ tendon could be inserted into the humerus while and at the same time restoring the native length/tension on the LHBT. The humeral fixation point was marked and freed from soft tissue with the use of electrocautery and soft tissue elevator. A 7-mm (female patient) or $8-\mathrm{mm}$ (male patient) reamer was used to create a unicortical bone tunnel. One limb of the suture was passed through the screwdriver and the appropriately sized PEEK (polyether ether ketone) tenodesis screw (Arthrex) was placed into the bone tunnel and advanced until it was flush with the anterior humeral cortex. The suture limbs were cut flush and the wound was irrigated and closed in a layered fashion.

Postoperatively, the operative extremity was immobilized in a sling for 2 weeks with full active and passive range of motion beginning immediately. Patients were restricted from performing resisted elbow flexion maneuvers for at least 6 weeks after surgery. Overhead strengthening and heavy lifting were delayed for approximately 3 months.

\section{Combined SLAP Repair and Biceps Tenodesis (SLAP-R+BT)}

Arthroscopy was again performed in standard fashion with identification and classification of the SLAP tear. In the presence of a SLAP II-IV tear leading to glenohumeral instability, as well as biceps tenosynovitis, a tenotomy was performed as described previously. Labral repair was then performed first, followed by subpectoral biceps tenodesis, both as described previously. Postoperatively, patients were instructed to follow the SLAP-R protocol previously outlined.

\section{Clinical and Functional Outcome Assessment}

Patient-reported outcomes scores, including the American Shoulder and Elbow Surgeons (ASES) score, Single Assessment Numerical Evaluation (SANE) score, Quick Disabilities of the Arm, Shoulder and Hand score, the General Health Short Form-12 physical component score, and patient satisfaction (scale 110, with 10 representing "very satisfied") were collected preoperatively and at final follow-up. Additional optional questions were administered to assess patients' participation in sports, both preoperatively and postoperatively. These questions evaluated the pain and functional ability to perform sports with the following: Pain with competition responses available were (1) no pain, (2) pain only after competition, (3) mild pain with competition, (4) moderate pain with competition, (5) severe pain with competition, and (6) pain prevents competition. Grade sport participation responses available were (1) equal to or above my preinjury level, (2) slightly below my preinjury level, (3) moderately below my preinjury level, (4) 
significantly below my preinjury level, (5) I cannot compete in my usual sport, and (6) I cannot compete in any sports. Ability to throw a ball 20 yards overhand responses available were (1) none, (2) mild, (3) moderate, and (4) severe. The minimal clinical important difference (MCID) and substantial clinical benefit (SCB) were the sum difference between preoperative and postoperative scores dichotomized as yes or no. Patient acceptable symptom state (PASS) was dichotomized yes or no if by the final postoperative scores. Failures requiring revision of SLAP lesion or biceps pathology treatment as well as postoperative complications were reported.

\section{Statistical Analysis}

All statistical analyses were performed with SPSS, version 11.0 (SPSS, Chicago, IL). Continuous numerical data are presented as mean \pm standard deviation and categorical values are presented as percentages. Data were tested for normal distribution using the Kolmogorov-Smirnov test. An independent or paired $t$ test was used for univariate analysis of normally distributed variables. For nonparametric data, the Mann-Whitney or Kruskal-Wallis tests were performed. The Wilcoxon signed-rank test for paired $t$ test was used to compare baseline and postoperative scores. The Mann-Whitney test also was used to compare patient-reported outcome (PRO) scores between 2 factors and the Kruskal-Wallis test used to compare PROs between the 3 treatment groups. Assuming nonparametric comparison, 2-tailed hypothesis testing, and an alpha level of 0.05 , group sizes of 21 and 28 are sufficient to detect an effect size of $d=0.85$ with $80 \%$ statistical power. Thus, we interpret that this study may be underpowered to detect group differences that are more subtle than $\mathrm{d}=0.85$. All $P$-values were 2 -tailed, and $P<.05$ was considered statistically significant.

\section{Results}

Seventy-two total patients met inclusion criteria (Fig 1). There were 38 shoulders in the BT group with 1 $(2.6 \%)$ shoulder requiring revision surgery, 13 in the SLAP-R group with no patient requiring revision, and 21 in the SLAP-R+BT group with $2(9.5 \%)$ shoulders requiring revision $(P=.356)$. A total of $67.6 \%$ of the patients had surgery on their dominant arm with $12.5 \%$ of all patients having had a previous SLAP repair (Table 1). Of the patients not undergoing revision, minimum 2-year follow-up was obtained for 32 of 37 $(86.4 \%)$ patients in the BT group, 11 of $13(84.6 \%)$ patients in the SLAP-R group, and 17 of $19(89.5 \%)$ in the SLAP-R+BT group. The mean age at surgery was 34.8 years (range, $14-45$ years) in 51 male and 21 female patients. Mean age at time of surgery was significantly different between the groups (36.5 BT vs 27.7 SLAP-R vs 36.5 SLAP-R+BT; $P=.003)$. Three total patients $(5.1 \%)$ of the BT and BT+SLAP groups required revision of their $\mathrm{BT}$ due to tenodesis failure or hardware irritation.

No significant difference was detected in PROs pre- or postoperatively between the treatment groups $(P>.05)$ using the Kruskal-Wallis test between each of the 3 groups (Table 2). Median satisfaction postoperatively was greatest in the SLAP-R+BT group at 10 of 10
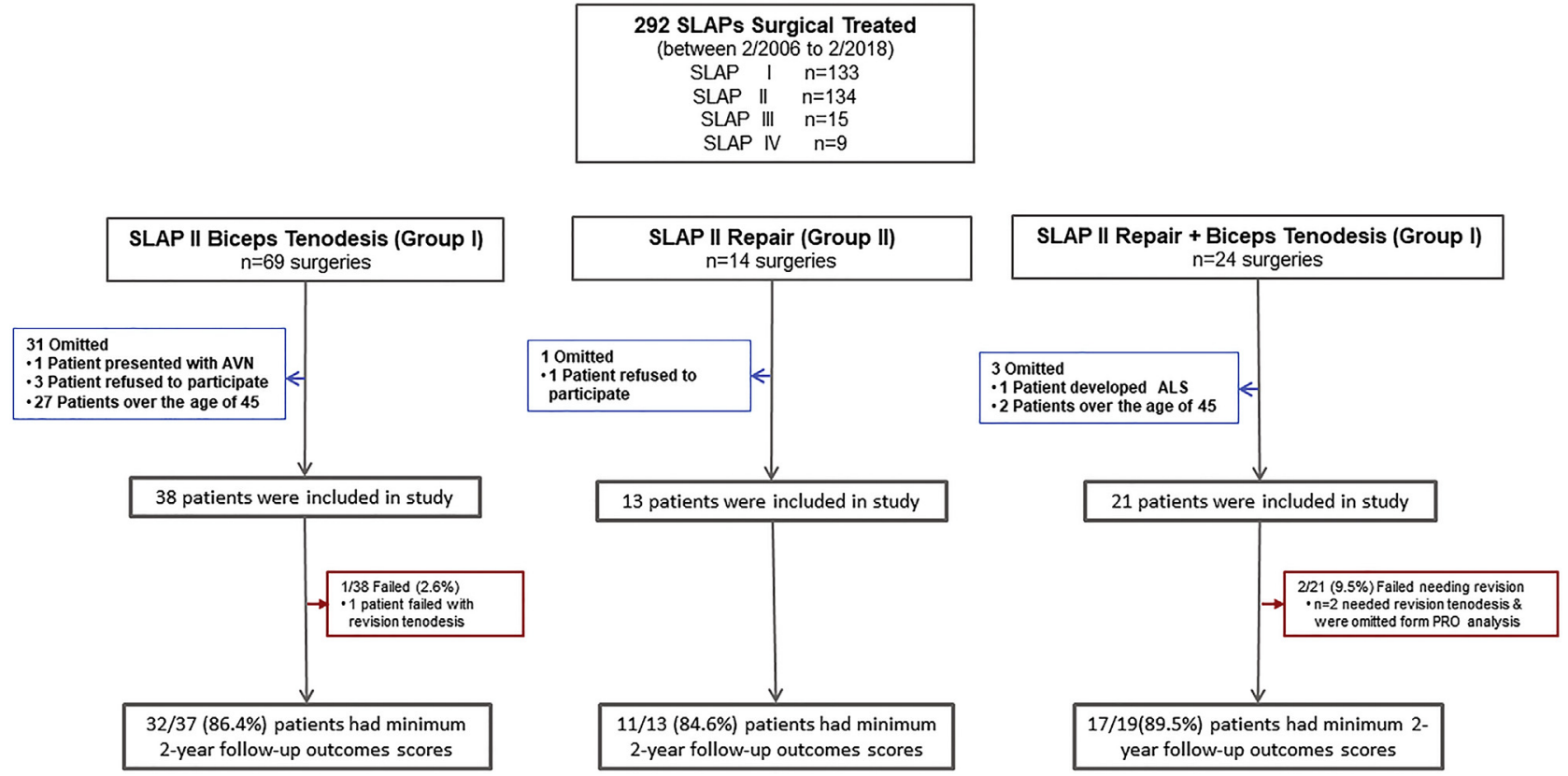

Fig 1. CONSORT (Consolidated Standards of Reporting Trials) flow diagram detailing inclusion and exclusion of patients through the study. (ALS, amyotrophic lateral sclerosis; AVN, avascular necrosis; PRO, patient-reported outcome.) 
Table 1. Demographic Data Demonstrating No Significant Differences Between Groups

\begin{tabular}{|c|c|c|c|c|}
\hline & BT & SLAP-R & SLAP-R+BT & $P$ Value \\
\hline Previous SLAP repair & $7(18.4 \%)$ & $2(18.2 \%)$ & None & .162 \\
\hline Age at sx, y & $36.5 \pm 7.3$ & $29.7 \pm 9.6^{*}$ & $35.9 \pm 7.4$ & .039 \\
\hline Sx on dominate arm & $25(65.8 \%)$ & $7(63.6 \%)$ & $14(66.7 \%)$ & .937 \\
\hline WC & $4(10.5 \%)$ & $1(9.1 \%)$ & $5(23.8 \%)$ & .261 \\
\hline LOA & $5(13.2 \%)$ & $1(9.1 \%)$ & $1(4.8 \%)$ & .792 \\
\hline DCE & $7(18.4 \%)$ & $1(9.1 \%)$ & $2(9.5 \%)$ & .596 \\
\hline Failures & $1(2.6 \%)$ & None & $2(9.5 \%)$ & .356 \\
\hline Years of postoperative follow-up & 3.6 (range $2-8$ ) & 4.6 (range $2-8$ ) & $3.5(2-7)$ & .296 \\
\hline
\end{tabular}

NOTE. SLAP-repair (SLAP-R) and SLAP-R+BT groups.

BT, biceps tenodesis; DCE, distal clavicle excision; LOA, lysis of adhesions; Sx, surgery; WC, worker's compensation.

${ }^{*}$ The SLAP-R group was statistically younger than the BT only, but no difference between BT and SLAP+BT groups. Statistically significant, $P<.05$.

(Table 2). Factors not significantly affecting postoperative PROs were SLAP grade and concomitant procedures, such as distal clavicle excision or lysis of adhesions $(P>.05)$. While PROs improved significantly from pre- to postoperatively for the BT and SLAP-R+BT groups, they did not significantly improve for the isolated SLAP-R group, with the exception of the General Health Short Form-12 physical component score (Fig 2). The BT and SLAP-R + BT groups showed significant improvement in return to sport pre- to postoperatively whereas the SLAP-R group did not (Table 3).

When we compared the SLAP-R group with the BT group, there was a statistical difference for the postoperative SANE scores $(P=.03)$. Otherwise, there were no significant differences in PROs between these 2 groups, with the $P$-values ranging from .597 to .988 . Additionally, there were no significant differences in PROs between SLAP-R and SLAP-R+BT for all PROs. The $P$ values ranged from .086 to .239 .

The percentage of each group meeting the MCID, SCB, and PASS ASES scores can be seen in Figure 3. These were set according to previously determined levels ( $>11$ points, $>16.8$ points, and $>86.2$ points, respectively). ${ }^{12,13}$ The SLAP-R+BT group consistently had the most patients reaching MCID, SCB and PASS, while the SLAP-R group consistently had the fewest. However, this was not statistically significant when comparing all 3 groups (Fig 3).

\section{Discussion}

The main findings of this study supported the hypothesis that SLAP II-IV lesions treated with BT or both SLAP-R+BT demonstrated improved outcomes compared with isolated SLAP-R at minimum 2-year follow-up. It was found that PROs improved significantly for both BT and SLAP+BT groups, but not for the isolated SLAP group. In addition, the BT and SLAP$\mathrm{R}+\mathrm{BT}$ groups showed significant improvement in return to sport pre- to postoperatively while the SLAP-R group did not. The BT group had significant improvement in postoperative SANE scores compared with the SLAP-R group. However, there was a greater revision rate in the BT and SLAP-R+BT groups than the SLAP-R group.

This study found results contradictory to previous literature. Chalmers et al. ${ }^{14}$ found that in 17 professional baseball players, BT alone resulted in return to play, defined as greater than 20 games, in 44\%, whereas only $25 \%$ of players who had SLAP + BT were able to return to play. These authors also found that high demand patients demonstrated worse outcomes following a combined SLAP and BT. ${ }^{10}$ Similar to our study, this study included a significantly older population in the isolated BT group. In addition, there were a significant number of worker's compensation patients within their combined group, which may have contributed to worse outcomes in this group. ${ }^{15}$ Our study found that PROs improved significantly for both

Table 2. Postoperative Patient-Reported Outcomes for BT

\begin{tabular}{|c|c|c|c|c|}
\hline & BT (Range) & SLAP-R (Range) & SLAP-R+BT (Range) & $P$ Value \\
\hline ASES score & $85.6(53.3-100)$ & $84.6(63.3-100)$ & $93.6(65-100)$ & .181 \\
\hline SANE score & $83.3(49-99)$ & $81.5(34-99)$ & $90.9(64-99)$ & .109 \\
\hline QuickDASH score & $13.7(0-47.7)$ & $15.0(0-34)$ & $7.2(0-29.5)$ & .122 \\
\hline Median satisfaction & $8(1-10)$ & $7(1-10)$ & $10(1-10)$ & .127 \\
\hline
\end{tabular}

NOTE. SLAP-repair (SLAP-R) and SLAP-R+BT groups.

ASES, American Shoulder and Elbow Surgeons; BT, biceps tenodesis; QuickDASH, Quick Disabilities of Arm, Shoulder and Hand; SANE, Single Assessment Numeric Evaluation; SF-12, General Health Short Form-12 Physical Component Score. 
BT Pre to Post PROs

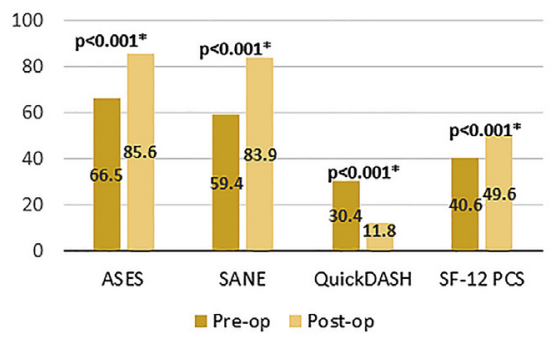

SLAP-R Pre to Post PROs

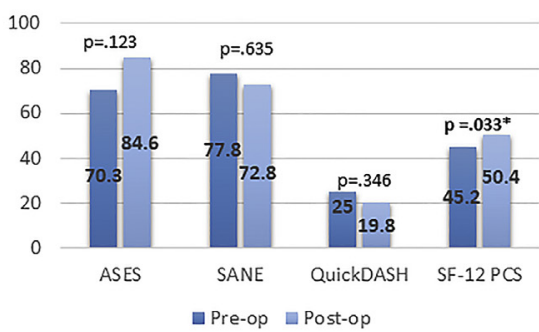

SLAP-R+BT Pre to Post PROs

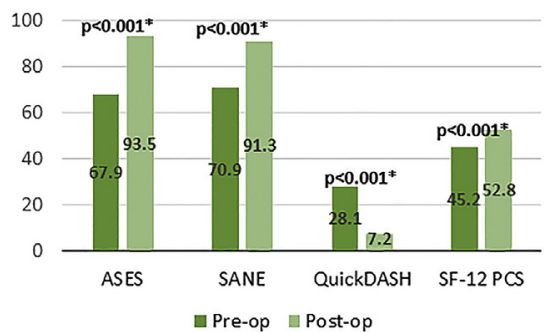

Fig 2. Pre- and postoperative patient-reported outcomes (PROs) for American Shoulder and Elbow Surgeons (ASES), Single Assessment Numeric Evaluation (SANE), Quick Disabilities of Arm, Shoulder, and Hand (QuickDASH), and Short Form-12 Physical Composite Scale (SF-12) for the biceps tenodesis (BT), SLAP repair (SLAP-R), and SLAP-R + BT groups. *Statistically significant, $P<.05$.

BT and SLAP+BT groups but did not for the isolated SLAP group. It is thought that the LHBT left intact can pull on the superior labrum and provide tensile forces at the SLAP repair site. In addition, the LHBT may have underlying pathology not appreciated by the surgeon, as it can occur more distal in the groove and may continue to act as a pain generator. ${ }^{16}$

The SLAP-R+BT group consistently had the most patients reaching MCID, SCB, and PASS for ASES score, whereas the SLAP-R group consistently had the fewest. However, with the numbers available, this was not statistically significant when comparing all 3 groups. This, again, was likely underpowered to find statistical significance. Moreover, the current utility of the MCID for patient-reported shoulder outcome instruments is limited by poor study methodology, inadequate reporting, and a lack of data. ${ }^{17}$ Further research is needed to define the MCID values more clearly for commonly used PROs in shoulder surgery. For this study, we assumed a PASS value of 86.2 in ASES, which was previously established in a study with patients undergoing arthroscopic rotator cuff repair. ${ }^{12}$
Another study established a PASS of 59 in ASES for patients undergoing $\mathrm{BT}^{13}$ However, the patients analyzed in this study started with greater ASES scores. There is no established PASS in ASES for SLAP-R or SLAP-R $+\mathrm{BT}$; therefore, we chose to use the value of 86.2 .

It has been shown that varying degrees of labral detachment from the superior glenoid and can result in increased translation and shear forces of the glenohumeral joint. ${ }^{1,2}$ Further, a recent cadaveric study demonstrated that a labral repair following biceps tenotomy restored glenohumeral stability to near baseline and provided increased resistive force to superior translation. ${ }^{18}$ In this study, there was a statistically different change in SANE scores pre- to postoperatively between isolated BT and isolated SLAP repair $(P=.033)$. In fact, postoperative SANE scores were worse, compared with preoperative scores, for the isolated SLAP repair group.

The results of this study demonstrate a clear benefit in ASES and other PROs for adding a BT to SLAP-R; however, combining procedures led to an increase in

Table 3. Pre- and Postoperative Return to Sport Outcomes

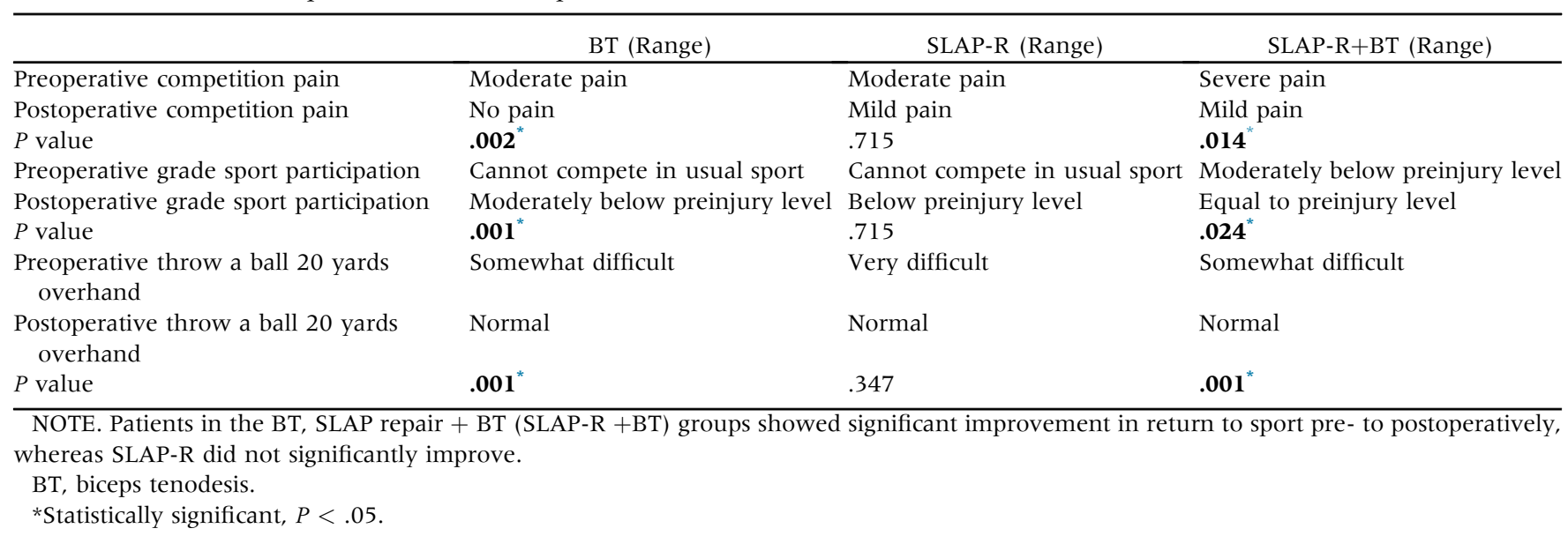


Fig 3. The percentage of each group meeting minimal clinical important difference (MCID), and patient acceptable symptom state (PASS) American Shoulder and Elbow Surgeons (ASES) score according to previously determined levels $(>11$ points, $>16.8$ points, and $>86.2$ points, respectively). ${ }^{13}$ (BT, biceps tenodesis; SLAP-R, SLAP repair.) substantial clinical benefit (SCB),

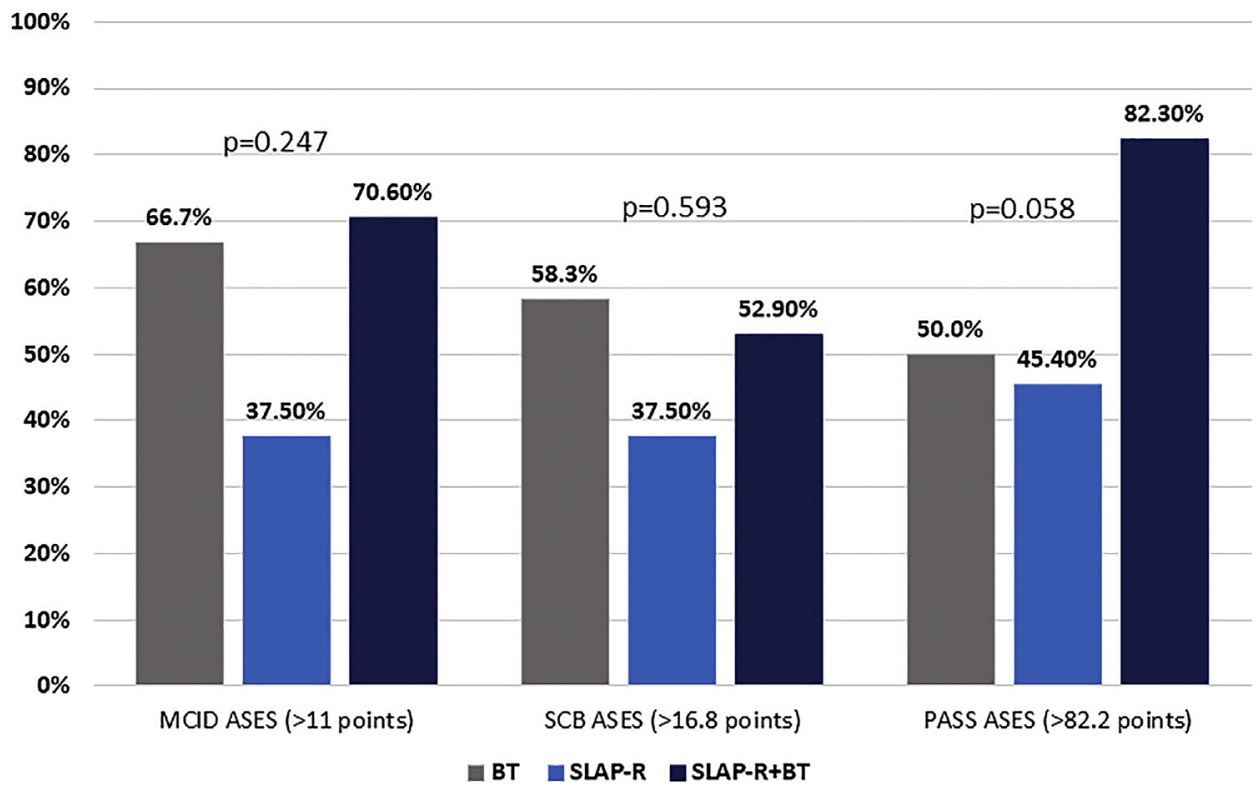

\section{Conclusions}

revision rate $(9.5 \%$ SLAP+BT vs $0 \%$ SLAP-R). The addition of a BT to a SLAP-R does introduce increased concern for complications regarding the BT. In our study, 3 total patients $(5.1 \%)$ of the BT and BT+SLAP groups required revision of their $\mathrm{BT}$ due to tenodesis failure or hardware irritation. It is important to acknowledge this increased risk but at the benefit of improved patient outcomes 2 years postoperatively.

\section{Limitations}

The potential limitations of this study are that it is a retrospective and nonrandomized study with a relatively small sample size. Revision SLAP repairs as well as those with concomitant debridement and distal clavicle excision were included in the study, which may lead to confounding variables and undue bias. In addition, there was a significantly younger population in the SLAP-R group, whereas the BT and SLAP+BT had the same mean age. This younger group may be more active or have greater expectations, leading to their decreased improvement of postoperative PROS. In this study, we particularly focused on isolated SLAP lesions, excluding patients with instability, rotator cuff lesions, or other shoulder pathologies. In addition, patients with a previous SLAP repair were included, which may introduce bias. Excluding these would have resulted in a significant reduction in the cohort size. The small number of included patients reflects the relative rarity of this condition in isolation and may not be generalizable to other populations. Future randomized controlled studies comparing SLAP-R with or without BT should be performed, with results stratified according to patient age and activity level.

\section{References}

1. Morgan CD, Burkhart SS, Palmeri M, Gillespie M. Type II SLAP lesions: Three subtypes and their relationships to superior instability and rotator cuff tears. Arthroscopy 1998; 14:553-565.

2. Patzer T, Habermeyer P, Hurschler C, et al. The influence of superior labrum anterior to posterior (SLAP) repair on restoring baseline glenohumeral translation and increased biceps loading after simulated SLAP tear and the effectiveness of SLAP repair after long head of biceps tenotomy. J Shoulder Elbow Surg 2012;21:1580-1587.

3. Boileau P, Parratte S, Chuinard C, Roussanne Y, Shia D, Bicknell R. Arthroscopic treatment of isolated type II SLAP lesions: Biceps tenodesis as an alternative to reinsertion. Am J Sports Med 2009;37:929-936.

4. Ek ET, Shi LL, Tompson JD, Freehill MT, Warner JJ. Surgical treatment of isolated type II superior labrum anterior-posterior (SLAP) lesions: Repair versus biceps tenodesis. J Shoulder Elbow Surg 2014;23:1059-1065.

5. Provencher MT, McCormick F, Peebles LA, et al. Outcomes of primary biceps subpectoral tenodesis in an active population: A prospective evaluation of 101 patients. Arthroscopy 2019;35:3205-3210.

6. Hurley ET, Fat DL, Duigenan CM, Miller JC, Mullett H, Moran CJ. Biceps tenodesis versus labral repair for superior labrum anterior-to-posterior tears: A systematic review and meta-analysis. J Shoulder Elbow Surg 2018;27:1913-1919. 
7. Nielen DVWM, Hammond J, Joyner PW. Biceps tenodesis vs. repair for type II SLAP tears in patients under 30 yearsold. Orthop J Sports Med 2017;5.

8. Sayde WM, Cohen SB, Ciccotti MG, Dodson CC. Return to play after Type II superior labral anterior-posterior lesion repairs in athletes: A systematic review. Clin Orthop Relat Res 2012;470:1595-1600.

9. Strauss EJ, Salata MJ, Sershon RA, et al. Role of the superior labrum after biceps tenodesis in glenohumeral stability. J Shoulder Elbow Surg 2014;23:485-491.

10. Chalmers PN, Monson B, Frank RM, et al. Combined SLAP repair and biceps tenodesis for superior labral anterior-posterior tears. Knee Surg Sports Traumatol Arthrosc 2016;24:3870-3876.

11. Snyder SJ, Karzel RP, Del Pizzo W, Ferkel RD, Friedman MJ. SLAP lesions of the shoulder. Arthroscopy 1990;6:274-279.

12. Cvetanovich GL, Gowd AK, Liu JN, et al. Establishing clinically significant outcome after arthroscopic rotator cuff repair. J Shoulder Elbow Surg 2019;28:939-948.

13. Puzzitiello RN, Gowd AK, Liu JN, Agarwalla A, Verma NN, Forsythe B. Establishing minimal clinically important difference, substantial clinical benefit, and patient acceptable symptomatic state after biceps tenodesis. J Shoulder Elbow Surg 2019;28:639-647.

14. Chalmers PN, Erickson BJ, Verma NN, D'Angelo J, Romeo AA. Incidence and return to play after biceps tenodesis in professional baseball players. Arthroscopy 2018;34:747-751.

15. Compton J, Glass N, Fowler T. The effect of workers' compensation status on the patient experience. JB JS Open Access 2019;4:e0003.

16. Murthi AM, Vosburgh CL, Neviaser TJ. The incidence of pathologic changes of the long head of the biceps tendon. J Shoulder Elbow Surg 2000;9:382-385.

17. Jones IA, Togashi R, Heckmann N, Vangsness CT Jr. Minimal clinically important difference (MCID) for patient-reported shoulder outcomes. J Shoulder Elbow Surg 2020;29:1484-1492.

18. Chan CM, Behrend C, Shields E, Maloney MD, Voloshin I. Effects of varying locations for biceps tendon tenotomy and superior labral integrity on shoulder stability in a cadaveric concavity-compression model. Arthroscopy 2014;30:1557-1561. 